\title{
Photodetectors in High Energy Physics
}

\section{Dieter Renker ${ }^{1}$}

Paul-Scherrer-Institute

5232 Villigen, Switzerland

E-mail: dieter.renker@psi.ch

\section{Abstract}

The needs of experiments in high energy physics have been for many decades the stimulus for detector developments. For calorimetry, particle identification with ring image Čerenkov detectors, time of flight measurements etc. sophisticated photo sensors have been realized. An overview will be given.

International workshop on new photon-detectors PD07

Kobe University, Kobe, Japan

27-29 June, 2007

\footnotetext{
$1 \quad$ Speaker
} 


\section{Introduction.}

The first scattering experiment in particle physics was based on the detection of photons. In 1906 Geiger, Marsden and Rutherford scattered alpha-particles on a thin gold foil and determined the scattering angle from the light flash produced at the impact point of the alpha-particles on a phosphor screen [1]. The light detector was the human eye which has a remarkable sensitivity when it is adapted to the dark.

In the year 1913 Elster and Geiter invented the photoelectric tube and in 1930 the first photomultiplier tube (PMT) was invented by L. Kubetsky [2]. In 1939 V. Zworykin and his colleagues from the RCA laboratories developed a PMT with electrostatic focusing [3], the basic structure of current PMT's, and a short time after it became a commercial product. Single photons were detectable from now on. Further innovations have led to highly sophisticated devices available nowadays. PMT's have two severe handicaps: They are very sensitive to magnetic fields (some devices tolerate axial magnetic fields) and their price is high because the complicated mechanical structure inside a vacuum container is mostly handmade.

Many modern experiments in high energy physics have calorimeters with high granularity inside strong solenoidal magnetic fields. This forced the search for an alternative to PMT's.

One of the simplest kind of photodiodes is the p-i-n photodiode in which an intrinsic piece of semiconductor is sandwiched between two heavily doped $n+$ and $p+$ regions [4]. This configuration produces a field which, even without an external field supplied, will tend to separate charges produced in the depleted region. The separated charges will be swept to the terminals and detected as current provided that they are not recombined. The smallest detectable light flash needs to consist of several hundred photons because the device has no internal gain and a charge sensitive amplifier is needed which introduces noise to the system. The p-i-n photodiode is used in most big experiments in high energy physics (CLEO, L3, BELLE, BABAR, GLAST ...).

Avalanche photodiodes (APD's) have internal gain which improves the signal to noise ratio but still some 20 photons are needed for a detectable light pulse. The excess noise factor, the fluctuation of the avalanche multiplication, limits the useful range of the gain. CMS is the first big experiment to use avalanche photodiodes [5].

In the Rockwell International Science Center, Stapelbroek et al. developed 1987 the Solid State PhotoMultiplier (SSPM). This is an APD with very high donor concentration which creates an impurity band $50 \mathrm{meV}$ below the conducting band. Later this device was modified to be less sensitive to infrared light and is now called Visible Light Photon Counter (VLPC). The small band gap forces an operation at very low temperatures of few degree Kelvin [6].

At the beginning of this millennium the Geiger-mode avalanche photodiode (G-APD) was developed $[7,8]$. This device can detect single photons like a PMT and therefore 
some people call it Silicon PhotoMultiplier, SiPM. The pulse height spectrum measured with a G-APD shows a resolution which is even better than what can be achieved with a photomultiplier and even with a hybrid photomultiplier tube (Fig. 1).

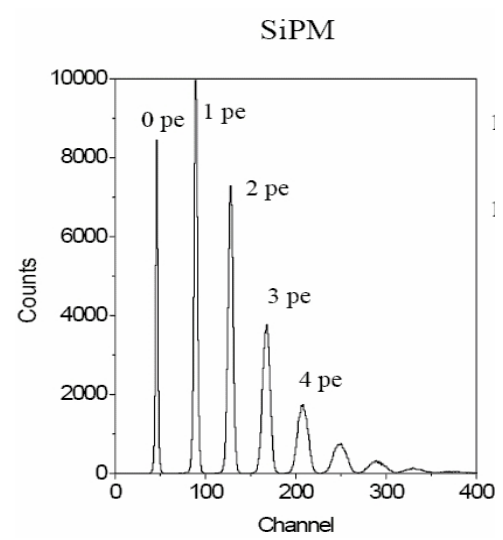

Fig. 1: Pulse height spectrum of weak light flashes measured with a G-APD. Taken from [9].

The development of gas filled detectors made enormous progress thanks to the invention of the Gas Electron Multiplier (GEM) [10]. In recent devices the photon feedback has been successfully suppressed and the ion back flow has been reduced to a very low level [11]. At moderate cost large area detectors can be built with submillimeter localization accuracy and a time resolution of better than $1 \mathrm{~ns}$. Gaseous detectors are sensitive to single photons in the spectral range from UV to visible light and the operation in high magnetic fields is possible. They have high gain (several $10^{5}$ ) and even so a long life time.

In the following chapters some recent detectors for high energy physics will be described. The problems that had to be dealt with and the solutions that have been found by selecting one of the above delineated light sensors will be pointed out.

\section{Calorimeters}

\subsection{Homogeneous calorimeters}

PMT's can be fabricated with large sensitive areas. The biggest are the tubes with 50 centimeter diameter used in the SuperKamiokande experiment.

The crystal ball for the measurement of pibeta decay $\pi^{+} \rightarrow \pi^{0}+\mathrm{e}^{+}+\mathrm{v}$ followed by $\pi^{0} \rightarrow \gamma+\gamma$, which has a branching ratio of $10^{-8}$, is made of 240 pure CsI crystals (Fig. 2). The crystal readout needs to cover the large area of the rear crystal face, it has to be very sensitive because the light yield of pure CsI is only few percent compared to $\mathrm{NaI}(\mathrm{Tl})$ and it has to be fast in order to allow an efficient suppression of accidental coincidences. The choice was a fast 10-stage PMT with $78 \mathrm{~mm}$ diameter from Electron Tubes (custom type 9822 QKB). The PMT's have a quartz entrance window (emission wavelength of pure CsI is $315 \mathrm{~nm}$ ) and are directly glued to the crystals [12]. 

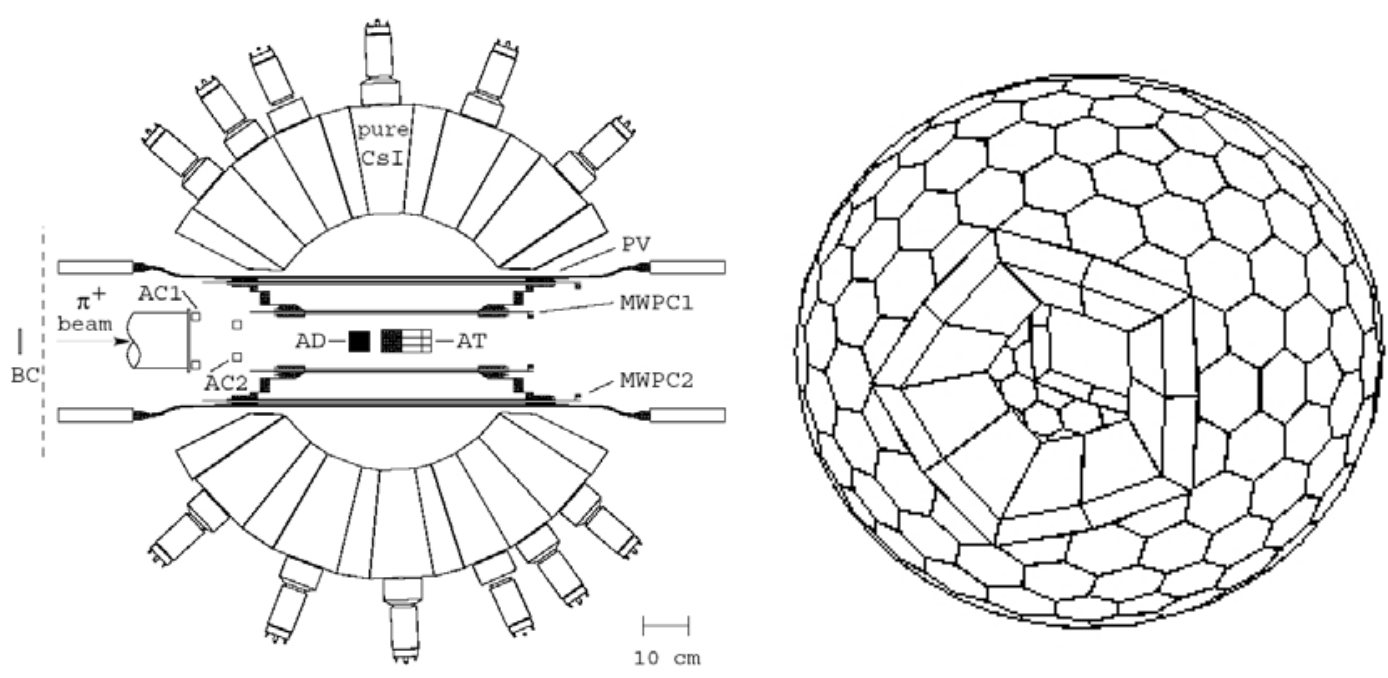

Fig. 2: Arrangement of pure CsI crystals in the so-called Pibeta experiment.

The amplification in the dynodes of a PMT has an extremely low level of noise. Summing over coincident signals from a large number of PMT's is therefore possible.

The so-called Muegamma experiment at PSI aims to measure the decay $\mu^{+} \rightarrow \mathrm{e}^{+}+\gamma$ with a sensitivity better than $5 \cdot 10^{-14}$. The $\gamma^{\prime}$ s will be detected in a 8001 liquid xenon calorimeter by the observation of the scintillation light with 800 PMT's. The deposited energy will be derived from the sum of all PMT signals and the position of the $\gamma$ conversion will be calculated from the distribution of the individual amplitudes [13].

In most general purpose detectors for experiments at accelerators with colliding beams all subsystems except the muon chambers are inside a solenoid with a strong magnetic field. The readout of the electromagnetic calorimeter has therefore to use a solid state photo detector. L3 with $\sim 12000$ BGO crystals was the first detector that employed p-i-n photodiodes [14]. Even more important was the selection of $\mathrm{p}-\mathrm{i}-\mathrm{n}$ photodiodes in calorimeters made of CsI(Tl) crystals because the emission of CsI(Tl) peaks at $550 \mathrm{~nm}$. Photodiodes have at this wavelength a quantum efficiency QE $~ 80 \%$ while PMT's have less than $10 \%$. Pioneered was the use of CsI(Tl) crystals and a p-i-n photodiode readout by CLEO [15]. The B-factories BELLE and BABAR have the same type of calorimeter and all achieve an energy resolution better than $2 \%$ for $1 \mathrm{GeV} \gamma$ 's.

Photodiodes have no gain. The operation is very stable but they need a charge sensitive amplifier which makes the signal rise time slow and introduces noise to the system $\left(\mathrm{C}_{\mathrm{PIN}} \sim 80 \mathrm{pF} / \mathrm{cm}^{2}\right)$. Calorimeters made of materials with low light yield (pure CsI in $\mathrm{KTeV}$ and Čerenkov calorimeters with lead glass) cannot use PIN photodiodes.

The full thickness of the p-i-n photodiodes $(300 \mu \mathrm{m})$ is sensitive. Charged particles (e.g. $\mathrm{e}^{+}$and $\left.\mathrm{e}^{-}\right)$which leak out at the rear end of the crystals and pass the diode produce an unwanted addition to the signal (Nuclear Counter Effect, NCE). A minimum ionizing particle creates some 100 electron-hole pairs per micron in silicon. This makes $\sim 30,000$ 
electron-hole pairs which fake additional energy in a calorimeter specially when the scintillation material has a relative low light yield, like $\mathrm{PbWO}_{4}$ [16].

The avalanche photodiode (APD) developed for the electromagnetic calorimeter of CMS has a thin p-doped layer of silicon in front of the p-n junction (Fig. 3) where the photo conversion takes place. The created electrons are amplified while the electrons produced by a MIP traversing the bulk (N, N- and N+ in fig.3) are not amplified. The contribution of the NCE can be evaluated by an effective thickness which is for the CMS APD $\sim 6 \mu \mathrm{m}$ [17], 50 times thinner than a p-i-n photodiode.

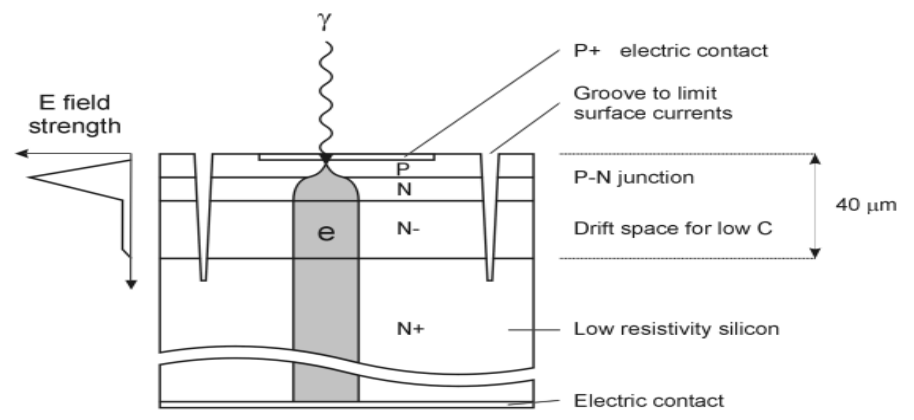

Fig. 3: Basic structure of the APD developed for CMS.

The internal gain of an APD improves the signal to noise ratio but the excess noise factor (avalanche fluctuations) and the gain sensitivity to voltage and temperature variations contribute to the energy resolution.

The energy resolution of CMS ECAL meets the design goal for the detector. For uniform illumination of a crystal with $120 \mathrm{GeV}$ electrons a resolution of $0.5 \%$ was achieved [18].

\subsection{Sampling calorimeters}

Almost a homogeneous calorimeter because of the relative small amount of absorber material is the KLOE calorimeter. It is made of 200 layers of scintillating fibers embedded in a lead absorber. For the readout fine mesh PMT's (Hamamatsu R5946) are used on both ends of the $430 \mathrm{~cm}$ long fibers. An excellent energy resolution of $5 \% / \sqrt{\mathrm{E}}(\mathrm{GeV})$ has been achieved. The time resolution of $54 \mathrm{ps} / \sqrt{\mathrm{E}}(\mathrm{GeV}) \oplus 50$ ps allows the determination of $\mathrm{K}^{0} \rightarrow \pi^{0}$, s vertices from the photon arrival time [19].

A real sampling electromagnetic calorimeter is Shashlik of LHCb. It has to stand a high radiation environment, has to have a good energy resolution, a fast response and a high dynamic range. Shashlik is a stack of scintillator and lead tiles with wavelength shifting fibers perpendicular to the tiles (Fig. 4) and with PMT readout (Hamamatsu 7899-20). The achieved energy resolution is $8.3 / \sqrt{ } \mathrm{E} \oplus 1.5 \% \%$ [20]. 


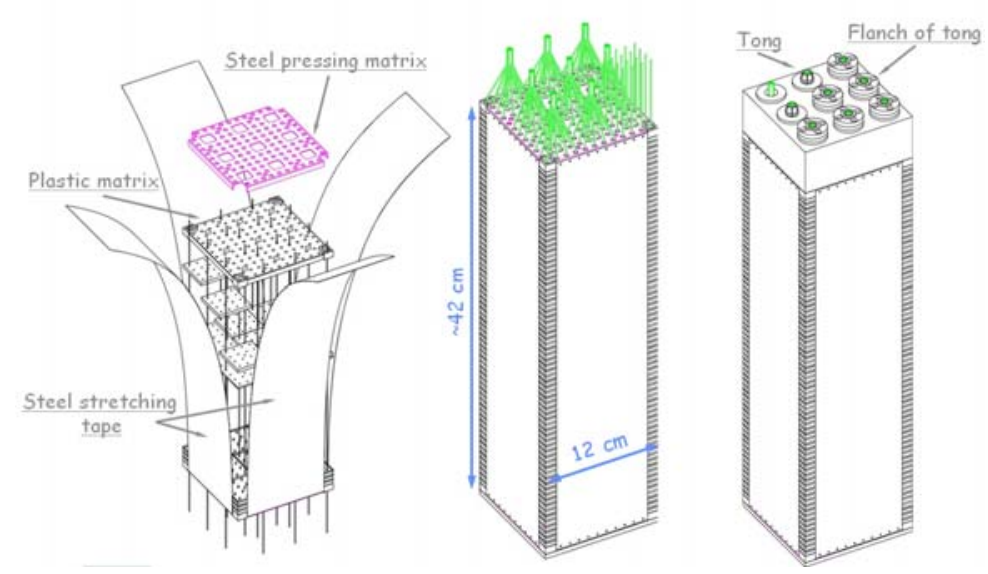

Fig. 4: Construction of the Shashlik electromagnetic calorimeter of LHCb.

Hadron calorimeters are almost always built as sampling devices because to some extent they allow to make the response to electrons equal to the response to hadrons ( $\mathrm{e} / \mathrm{h}=1$, compensation) which is crucial for the energy resolution. The best example is the ZEUS calorimeter with uranium and scintillator plates [21].

The energy sampled is typically a few percent of the total incident energy. A small number of photons have to be detected with a good signal to noise ratio. Up to now all sampling hadron calorimeters which are based on the detection of light from plastic scintillators use PMT's.

The hadron calorimeter of CMS is made of brass, plastic scintillator tiles, wavelength shifting fibers and proximity focused hybrid PMT's. It needs to work in a $4 \mathrm{~T}$ magnetic field, has to have a large dynamic range, high position sensitivity and low crosstalk $[22]$.
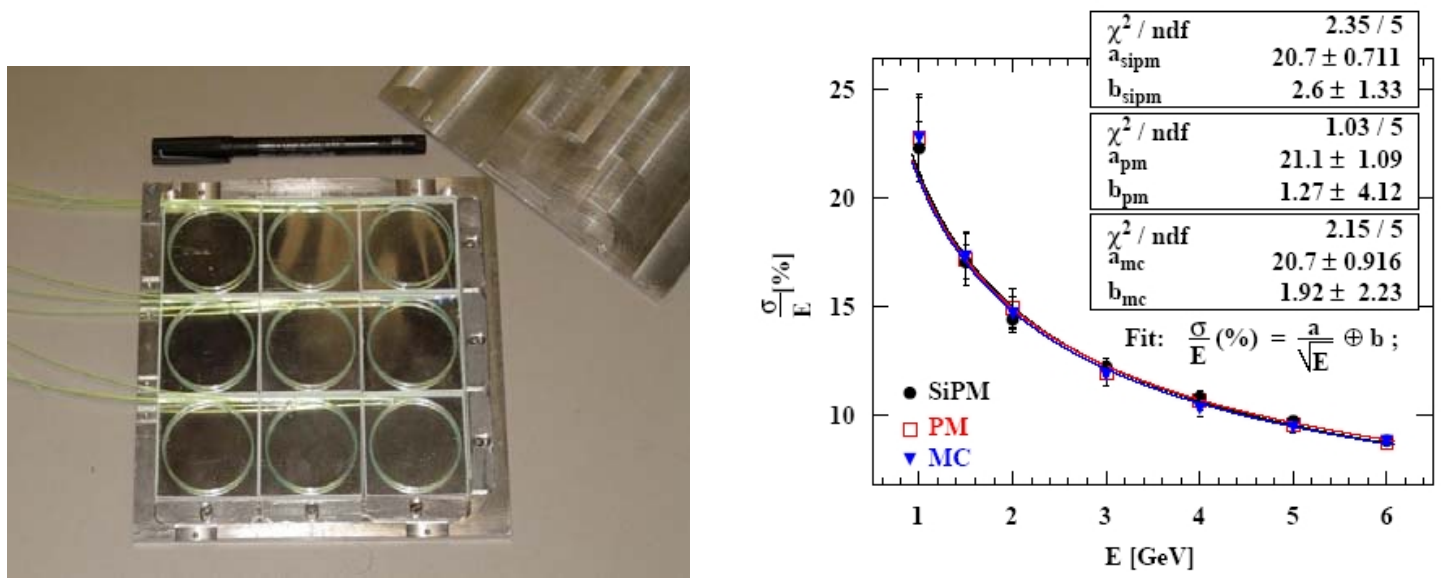

Fig. 5: Left: Tiles with wavelength shifting fibers. Right: Measured energy resolution with Geiger-mode APD's (solid points), multi anode PMT's (squares) and MC prediction (triangles).

The physics requirements at the International Linear Collider (ILC) impose strict demands on the performance of calorimeters. The ultimate goal is to achieve a jet 
energy resolution of about $30 \% / \sqrt{ } \mathrm{E}$ in order to increase the sensitivity for reconstruction of the W, Z and Higgs bosons and supersymmetric particles. The CALICE collaboration is presently constructing a test hadron calorimeter with 7620 scintillator tiles readout by the novel Geiger-mode APD (other names are SiPM, MPPC, MRS-APD ...). This prototype (Fig. 5) is the first device which uses Geiger-mode APD's on a large scale [23].

\section{Ring Image Cherenkov Counter (RICH)}

RICH counters measure the photon impact point on the photon detector surface. Needed is detection of single photons with large area coverage (up $11 \mathrm{~m}^{2}$ ), good spatial resolution, high efficiency and good signal-to-noise ratio. Individual RICH counter have in addition special requirements: operation in (high) magnetic fields, high rate capability, very high spatial resolution and excellent timing (time-of-arrival information and background reduction). Usually the available space is very limited and the material budget has to be very low to reduce the scattering and energy loss in front of calorimeters.

The classical example is the RICH of CLEO. It has a LiF radiator, an expansion volume and a wire chamber filled with a photo sensitive gas (methan and triethylamine, TEA) [24]. More recent RICH detectors use CsI to convert Cherenkov photons to free electrons. The CsI is deposited on the cathode pads of a wire chamber (ALICE [25], HADES [26], COMPASS [27]) or on the first foil of a triple Gas Electron Multipler (GEM) like in the PHENIX RICH [28] (Fig. 6).
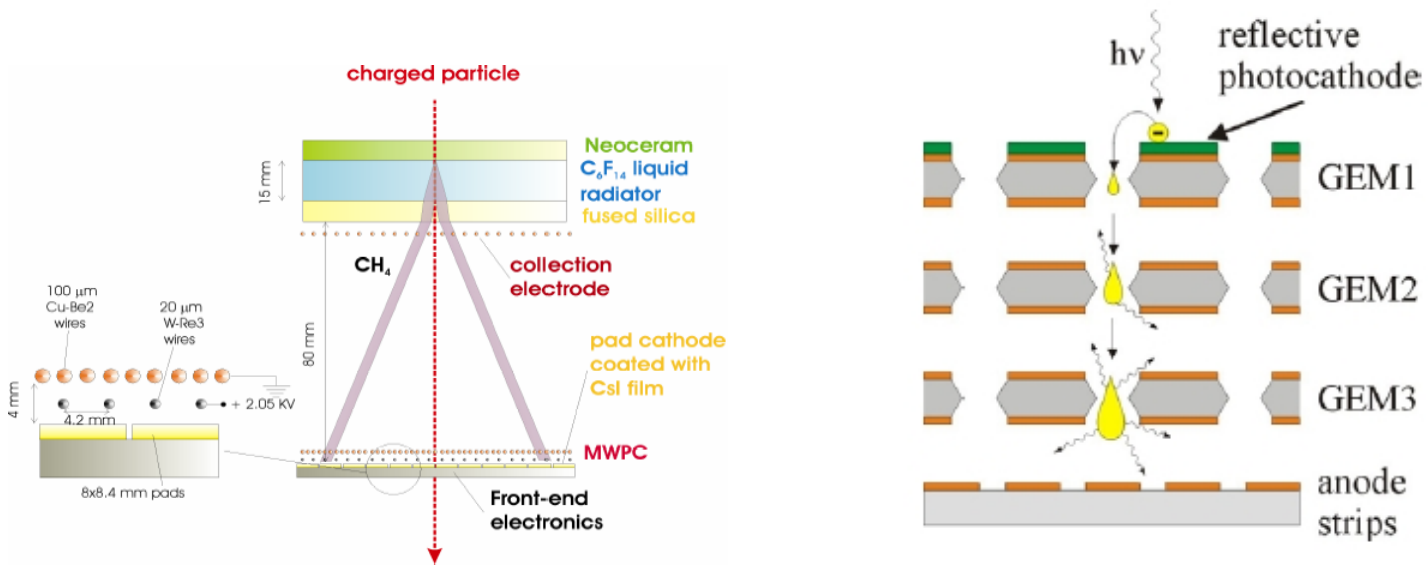

Fig. 6. Construction principle of the ALICE (left) and PHENIX RICH (right).

In the Compass experiment the central region of the RICH counter was recently upgraded to enable running at higher beam intensities, higher trigger rates and with a nanosecond time resolution for background suppression [27]. The wire chamber photon detector was replaced by an array of multianode PMTs very similar to the HERA-B RICH [29]. The PMT's are Hamamatsu 16 channel tubes (R5900-M16).

The RICH for $\mathrm{LHCb}$ has to cover a large area $\left(2.8 \mathrm{~m}^{2}\right)$ with high active area fraction. It needs to be fast compared to the $25 \mathrm{~ns}$ bunch crossing time, has to operate in a small 
magnetic field and has to have a granularity of $2.5 \times 2.5 \mathrm{~mm}^{2}$. A hybrid PMT with $5 \mathrm{x}$ demagnification has been chosen with an anode made of a pixel detector with 8192 channels organized in 1024 super-pixels of $500 \times 500 \mu \mathrm{m}^{2}$ size [30].

A very compact particle identification can be achieved with the Detection of Internally Reflected Cherenkov light (DIRC). The BABAR DIRC is based on the detection of Cherenkov photons trapped in a $5 \mathrm{~m}$ long quartz radiator bar (Fig. 7). The patterns on the photon detectors (PMT's ETL 9125) are complicated but result in well resolved peaks in the angle distribution [31].
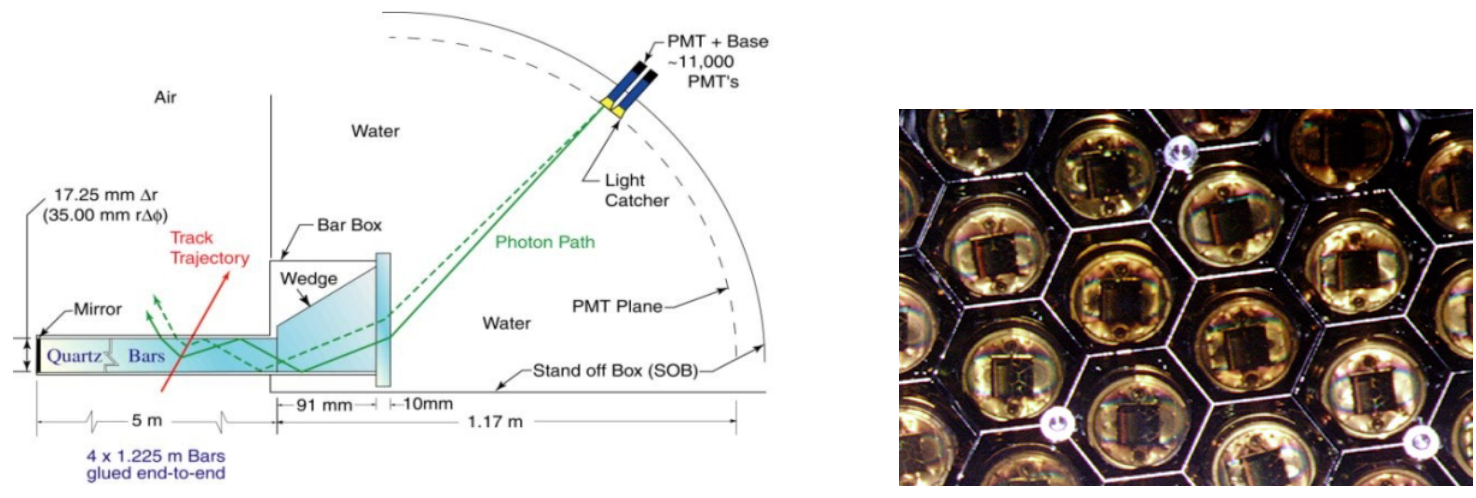

Fig. 7: Principle of the BABAR DIRC (left) and a view of the PMT array for the readout (right).

\section{Time of Flight detectors (TOF)}

Particle identification at relative low energies is done in the BELLE detector by TOF. 128 plastic scintillators ( $3 \mathrm{~m}$ long, $4 \times 6 \mathrm{~cm}^{2}$ ) with fine mesh PMT's (Hamamatsu R6680) on both sides of the TOF detector provide a time resolution of less than $100 \mathrm{ps}$ and allow a $\pi / \mathrm{K}$ separation up to $1.2 \mathrm{GeV}$ [32]. An Aerogel Cherenkov Counter (ACC) extends the $\pi / \mathrm{K}$ separation to $3.5 \mathrm{GeV}$. It also utilizes fine mesh PMT's.

\section{Tracking}

Scintillating fiber tracking technology has demonstrated great potential for high speed charged particle tracking and triggering. The D0 tracker consists of 80000 fibers, $2.5 \mathrm{~m}$ and $1.7 \mathrm{~m}$ long. The light produced by charged particles crossing a fiber is transported via $\sim 10 \mathrm{~m}$ long optical fibers to Visible Light Photon Counters (VLPC's) housed in a cryostat which regulates the operation temperature (6 to 8 degree Kelvin). Few photons arrive at the photo detector which therefore needs to have high QE of some $80 \%$ and single photon detection capability [33].

The target tracker of the OPERA experiment covers an area of $3000 \mathrm{~m}^{2}$. Its purpose is to locate the lead/emulsion brick where a neutrino interaction occurred [34]. In total 31744 scintillator strips $\left(1 \times 2.6 \times 686 \mathrm{~cm}^{3}\right)$ with a wavelength shifting fiber in a groove are grouped in 496 modules (64 strips/module). The 64 fibers of each module are on 
both sides connected to 64 channel multi anode PMT's (Hamamatsu H 7546). The construction is similar to the tracker in the MINOS experiment [35].

\section{Conclusion}

A large variety of techniques of photo sensors has been developed and integrated in present detectors for high energy physics. There is an almost infinite number of alternative designs which have been proposed and partly tested in prototypes. The working horse is still the PMT when weak light flashes need to be detected. New types of photo sensors are always quickly being adopted in high energy physics experiments. The new Geiger-mode avalanche photodiodes will for sure have a heavy impact on the design of future detectors.

\section{References}

[1] E. Rutherford, Philosophical Magazine, Series 6, 21 (1911) 669

and H. Geiger and E. Marsden, Proceedings of the Royal Society 82A (1909) 495.

[2] B.K. Lubsandorzhiev, Nucl. Instr. Meth. A 567 (2006) 236.

[3] V.K. Zworykin and J.A. Rajchman, Proc. IRE 27 (1939) 558.

[4] K. Yamamoto et al., Nucl. Instr. Meth. A 253 (1987) 542.

[5] G. Alexeev et al., Nucl. Instr. Meth. A 385 (1997) 425.

[6] A. Bross et al., Nucl. Instr. Meth. A 477 (2002) 172.

[7] P. P. Antich et al., Nucl. Instr. Meth. A 389 (1997) 491.

[8] V. Saveliev and V. Golovin, Nucl. Instr. Meth. A 442 (2000) 223.

[9] P. Buzhan et al., Nucl. Instr. Meth. A 504 (2003) 48.

[10] F. Sauli, Nucl. Instr. Meth. A 386 (1997) 531.

[11] A. Breskin et al., Nucl. Instr. Meth. A 553 (2005) 46.

[12] E. Frlež et al., Nucl. Instr. Meth. A 526 (2004) 300.

[13] A. Baldini et al., Nucl. Instr. Meth. A 545 (2005) 753.

[14] J.A. Bakken et al., Nucl. Instr. Meth. A 254 (1987) 535.

[15] E. Blucher et al., Nucl. Instr. Meth. A 249 (1986) 201.

[16] J. P. Peigneux et al., Nucl. Instr. Meth. A 378 (1996) 410.

[17] Th. Kirn et al., Nucl. Instr. Meth. A 387 (1997) 199.

[18] P. Adzic et al., CMS NOTE 2006/148.

[19] A. Antonelli et al., Nucl. Instr. Meth. A 370 (1996) 367.

[20] B. Perret, Nuvl. Phys. B 142 (2005) 442.

[21] M. Derrick et al., Nucl. Instr. Meth. A 309 (1991) 77.

[22] P.Cushman et al., Nucl. Instr. Meth. A 442 (2000) 289.

[23] V. Andreev et al., Nucl. Instr. Meth. A 540 (2005) 368.

[24] M. Artuso et al., Nucl. Instr. Meth. A 409 (1998) 375.

[25] A. Gallas et el., Nucl. Instr. Meth. A, in press.

[26] L. Fabbietti et al., Nucl. Instr. Meth. A 502 (2003) 256.

[27] P. Abbon et al., Nucl. Instr. Meth. A 572 (2007) 419.

[28] I. Ravinovich et al., Nucl. Instr. Meth. A 774 (2006) 903.

[29] I. Ariño et al., Nucl. Instr. Meth. A 516 (2004) 445.

[30] S. Amato et al. (LHCb collaboration), CERN LHCC 2000-037.

[31] I.Adam et al., Nucl. Instr. Meth. A 538 (2005) 281. 
[32] E. Nakano, Nucl. Instr. Meth. A 494 (2002) 402.

[33] B. Baumbaugh et al., Nucl. Instr. Meth. A 345(1994) 271.

[34] T. Adam et al., Nucl. Instr. Meth. A 577 ( 2007) 523.

[35] N. Tagg et al., Nucl. Instr. Meth. A 539 (2005) 668. 